\title{
Non-lieu et utopie : la diaspora chinoise et le territoire
}

\author{
Emmanuel MA MUNG *
}

RÉSUMÉ.- Deux caractères morphologiques objectifs définissent une diaspora : la multipolarité de la migration et l'interpolarité des relations. À ceux-ci s'ajoute une autre caractéristique : la perception d'exterritorialité, forme particulière de représentation de soi dans l'espace. Elle suppose une prise de conscience de la configuration spatiale de la diaspora et le développement d'une culture, conduisant à optimiser les ressources spatiales. La migration internationale chinoise se voit progressivement comme une diaspora. La position d'exterritorialité, la perception de ce non-lieu, de cette a-topie est une étape indispensable comme intellectualisation du fait morphologique de la diaspora pour établir et améliorer son propre fonctionnement, lui permettant de concevoir cette sorte d'utopie qui consiste à rêver de soi-même dans une vie hors du territoire.

\section{CHINOIS, DIASPORA, EXTERRITORIALITÉ, MIGRATION, RÉSEAU}

\begin{abstract}
A-topia and utopia : the Chinese diaspora and territory.- Diaspora is defined by two objective morphological characteristics, multipolar migration and interpolar relationships. A third characteristic can be add ed to these : perception of extraterritoriality, which is a specific form of spatial representation of self. This implies an awareness of the diaspora 's spatial configuration and culture leading to optimisation of its spatial resources, i.e. multipolarised migration and interpolarised relationships. The Chinese who have emigrated begin gradually to see themselves as a diaspora. The situation of extraterritoriality and the perception by a diaspora of a-topia - non-place contribute to generating and improving the diaspora's own operation as a diaspora, which in its turn enables the kind of utopia that consists in dreaming of oneself as living a life outside of territory.
\end{abstract}

\section{CHINESE, DIASPORA, EXTRATERRITORIALITY, MIGRATION, NETWORKS}

"Un très ancien poème chinois dit approximativement : "Partout où l'océan pousse ses vagues, accostent les Chinois". Les migrations ultramarines sont donc inscrites depuis fort longtemps dans l'univers chinois ${ }^{1}$. Elles commencèrent dans la périphérie méridionale, le Nan Yang, et orientale, Taiwan, puis s'élargirent aux constellations insulaires océanes indiennes et

\footnotetext{
* Chargé de Recherche au CNRS, MIGRINTER, URA 1145, département de géographie, Poitiers

${ }^{1}$ Parmi les nombreuses publications sur l'aspect historique des migrations chinoises en Asie du Sud-Est voir surtout : P. TROLLIET, G. WANG, M.K. TSAI et le classique V. PURCELL ; à titre anecdotique E. DENNERY et R. DESCHAMPS.
} 
pacifiques» $^{2}$. Elles abordèrent ensuite les rives orientales du Pacifique, c'est-à-dire les franges extrême-occidentales des Amériques. Et cette inversion toponymique orient-occident, liée à la circularité du Monde, cette désignation paradoxale d'un même lieu par un mot et son contraire, cette impossibilité de localiser un lieu dans une sorte d'en-soi topologique est déjà constitutive d'une utopie au sens d' une a-topie sémantique, puisqu'un lieu concret est, dans la langue naturelle, à la fois ici et ailleurs, mais un ailleurs symétrique. Ailleurs est pourtant ici. Cette fulgurance des lieux géographiques peut être le point de départ pour s'interroger sur le rapport de la diaspora au territoire ${ }^{3}$.

Une des questions à poser à propos de la diaspora peut être celle-ci : comment une société tient-elle malgré la distance ? Car, plus que pour tout autre, la question de la distance intervient dans l'organisation de cette société. À partir de cette question géographique et de sa déclinaison en de multiples autres sur l'organisation économique ou l'évolution historique, on est conduit à s'interroger sur les rapports que la diaspora entretient avec le territoire dans cette situation particulière d'une dissémination à l'échelle du Monde. Bien qu 'il y ait une grande variété de situations, nous voudrions suggérer que liée aux formes objectives de la diaspora, apparaît une forme particulière de représentation de soi dans l'espace, caractérisée par une position d'exterritorialité ${ }^{4}$. C'est cette forme paradoxale de rapport au territoire que nous essaierons de décrire et dont nous examinerons quelques effets ${ }^{5}$, en considérant les diasporas comme des corps sociaux qui mettent en oeuvre des stratégies qui leur permettent de subsister en tant qu'entités sociales distinctes, différentes, identifiant les autres dans le mouvement de leur identification à elles-mêmes.

\section{I - Construction de l'exterritorialité}

Deux caractères morphologiques objectifs définissent à notre sens une diaspora : la multipolarité de la migration (ou dispersion) et l'interpolarité des relations avec le pays d'origine et entre les différents pôles de la migration ${ }^{6}$, qui se traduit non seulement par des visites et des relations d'affaires entre les différents pôles, mais aussi par des migrations d' un pôle à l'autre. Ainsi de ce Chinois teochew du Cambodge, dont les ascendants sont originaires de la province cantonaise : réfugié en France et installé dans les années 1970 à Paris, il émigre durant plusieurs années au Canada, d'abord à Montréal puis à Vancouver, revient à la fin des années 1980 en France, où il ouvre un restaurant et un commerce d'objets exotiques à Rouen grâce à ses gains canadiens ainsi qu 'à des emprunts auprès de compatriotes teochew et wenzhou (province du Zhejiang) parce qu'il estime que les perspectives commerciales sont devenues meilleures en France qu'au Canada. Outre la migration entre différents pôles d'implantation de la diaspora chinoise, ce cas montre que les relations s'élargissent à des groupes chinois d'origines et de dialectes différents. De nombreux autres exemples ont dévoilé les itinéraires internationaux et les implantations successives de migrants dans l'espace de la diaspora, par exemple de l'Amérique latine vers l'Amérique du Nord (Daniel Bao, 1992 ; Lok Siu, 1992) ou vers les Caraïbes (Ramon A. Mon, 1992).

\footnotetext{
2 Éditorial du numéro de la Revue européenne des migrations internationales consacré à la diaspora chinoise en Occident, vol. 8, n³, 1992.

${ }^{3}$ Voir M. BRUNEAU (1994) et A. MÉDAM (1991).

${ }^{4}$ Sur la localisation mondiale, voir P. TROLLIET (1994), D.L. POSTON et M.Y. YU (1990).

${ }^{5}$ Voir E. MA MUNG 1991, 1992 a, 1994. Un premier cadre conceptuel et de problématisation du dispositif économique et des ressources spatiales d'une économie de diaspora est donné dans E. MA MUNG (1992 b).

${ }^{6}$ Pour plus de détail sur la multipolarité de la migration et l'interpolarité de la migration, voir: E. MA MUNG (1992 b).
} 
Ces multimigrations se font toujours dans l'espace de la diaspora ; l'état général de l'économie compte plus que la situation de l'emploi dans le choix du pays d'immigration, car il ne s'agit pas d'une migration de main-d'oeuvre au sens classique, mais d'une migration qui s'envisage comme " entrepreneuriale » même si elle est souvent migration de travail à ses débuts, et orientée vers les entreprises chinoises du pays d'immigration. La segmentation nationale de l'espace de la diaspora n'a de sens que selon les différences de réglementations, de cultures, de potentialités économiques : elle signale des occasions de migrer et de circuler. Les territoires nationaux apparaissent d'abord dans l'espace transnational de la diaspora comme des espaces de plus ou moins grande circulation.

Une autre caractéristique nous semble essentielle : la perception d'exterritorialité, qui est une forme particulière de représentation de soi dans l'espace ${ }^{7}$. Ce qui caractérise la diaspora, c'est l'arrachement du territoire d'origine ${ }^{8}$, l'impossibilité de se reproduire dans un espace physique clos, circonscrit et tangible dont elle procéderait traditionnellement "naturellement », notamment lorsque la migration a perdu son caractère temporaire, comme la migration chinoise $^{9}$. Elle se reproduit alors dans un espace imaginaire, fantasmé, reconstruit à l'échelle internationale. L'identification nationale-territoriale est transcendée en une vision de soi dans une sorte d'exterritorialité : cette perception, ce sentiment assurent le lien de la diaspora. Cette conscience de soi dans un non-lieu est une autre différenciation par rapport aux autres groupes migrants. Dans une certaine mesure, on pourrait dire qu'il y a diaspora quand il y a conscience de la diaspora. La diaspora serait d'abord un sentiment, une utopie, un rêve de soi-même. Mais c'est à partir de cette utopie que se construit effectivement la diaspora. C'est cette conscience qui lui permet de pousser des ramifications et de développer l'écheveau de ses réseaux.

Cette expérience particulière qui renforce le caractère diasporique conduit à faire valoir le territoire non plus comme espace unique, irremplaçable, d'où l'on procède et qui par conséquent fonde l'identité, mais comme espace pouvant équivaloir à d'autres. Équivalent, il peut s'échanger contre d'autres; de ce fait, on peut se mouvoir sans se désincarner dans les autres espaces ; un parcours est sentimentalement possible de l'un à l'autre. Cette équivalence est renforcée lorsque le territoire d'origine (l'Asie du Sud-Est pour certains Chinois) est différent du lieu mythique d'origine. La diaspora sait intuitivement et progressivement ce par quoi elle se construit idéologiquement comme diaspora, que son territoire n'est pas un lieu précis, mais une multitude d'équivalents; aucun n'est un lieu irremplaçable de l'identité. Le territoire est partout, donc nulle part. De l'équivalence des lieux, on peut déduire que la perception d'exterritorialité est une entreprise de réification de l'espace, en même temps que cette dernière est la condition de la position d'exterritorialité.

\footnotetext{
${ }^{7}$ Voir aussi S.M. LYMAN (1974, p.7) mais il s'agit là d'une territorialité culturelle et institutionnelle par séparation de la société d'accueil - selon l'auteur -, assez différente de celle que nous envisageons.

${ }^{8}$ Cet arrachement peut avoir une origine violente et prendre la forme d'une expulsion, comme pour la diaspora juive ou arménienne. Il peut être aussi causé par une autre forme de violence telle que la désagrégation sociale dans les régions d'origine et la misère, comme pour les Chinois ou les Libanais au XIXc siècle. Cf. E. MA MUNG. et A. ABDULKARIM (1991).

${ }^{9}$ Les retours définitifs restent faibles par rapport à la masse vivant hors de Chine: 25 à 35 millions d'individus, selon les comptages répartis entre plus de 130 pays. Les visites en revanche se multiplient, et les relations économiques se développent de façon considérable. Cf. P. TROLLIET (1994) et de nombreux articles de presse cités dans la bibliographie. Sur la réalité des investissements des Chinois d'outre-mer en Chine continentale voir SHU YAN : «Quand les milliards de dollars d'investissements ne sont qu 'un trompe-l'oeil ». Zhenming, janvier 1993, p. 24-25 ; repris par l'excellente revue française éditée à Hong Kong, Perspectives Chinoises, n' 11-12, janvier, février 1993.
} 


\section{Mythification et mystification de la terre d'origine}

Le territoire du pays d'origine représente toujours un idéal dans la mémoire de la diaspora : d'où la puissance des références et la mythification du pays d'origine dans l'imaginaire de la diaspora ${ }^{10}$. Pourtant, elle sait confusément que son identité ne s'origine plus là-bas, et qu'elle se construit dans le mouvement même de la dispersion sur ce territoire inimaginable et inlocalisable parce que possible partout. La diaspora n'est donc pas tout à fait dupe de la mystification que constitue pour elle la terre d'origine dans la construction de son être en migration. Et cela renforce d'autant son sentiment d'exterritorialité.

Au fond, la diaspora apprend peu à peu que son identité est surtout et partout localisée dans le vaste espace qu'elle parcourt, dans un territoire impensable en raison de son étendue. Elle est contrainte de suspendre son identité dans une sorte d'éther supranational, dans un territoire inimaginable et donc imaginaire parce que désiré, convoqué, mais jamais réalisé. Ainsi se pose de façon implicite la question de la localisation de l'identité et du rapport entre identité et lieux. Des éléments de réponse sont fournis par Alain Tarrius (1994) qui, envisageant les populations de migrants à partir de son " paradigme mobilitaire " et relisant Maurice Halbwachs, souligne que, pour ces populations, « c'est accrocher tous les lieux parcourus par soi-même et les autres que l'on reconnaît comme identiques, à une mémoire qui, devenue collective, réalise une entité territoriale ». Cette entité que l'auteur considère comme territoriale correspond dans une certaine mesure à notre non-lieu.

\section{Processus de mise en place de l'exterritorialité}

Comment, à partir de ce rapport particulier au territoire physique que constitue l'absence de relation essentialiste à celui-ci, la diaspora se construit-elle une représentation d'elle-même dans ce qu 'elle voudrait être un rapport au territoire-Monde ? Ce rapport est paradoxal en raison de l'impossibilité progressive pour la diaspora d'identifier son territoire car celui-ci devient, au fur et à mesure et idéalement, l'ensemble du globe. La diaspora émerge de la conscience qu'elle prend de sa dispersion. C'est en reconnaissant peu à peu les lieux où elle se déploie qu'elle prend conscience d'elle-même et qu'elle peut se représenter l'espace physique sur lequel elle se déploie. Mais celui-ci est-il pour autant son territoire ?

Jusqu'à ce moment, l'espace que ses membres perçoivent n'est que la localité dans laquelle ils se trouvent, opposée au pays d'origine : un espace migratoire classique. Dans la prise de connaissance puis de conscience des lieux où se trouvent d'autres semblables, adviennent deux phénomènes qui construisent la diaspora et renforcent sa conscience. La possibilité de migrer dans des espaces semblables, qui ne sont pas tout à fait les mêmes mais pas tout à fait d'autres. Chaque localité n'est différenciée des autres que par les conditions de sa localisation, ce qui est une autre expression de l'équivalence des lieux. Dans cette vision qu'elle a d'ellemême et de son déploiement spatial survient aussi la conscience de l'impossibilité de la territorialisation, d'un territoire tangible et définitif, aux limites simples, repérables aisément. Elle se voit dans un arrachement, un décollement du territoire, d'où le sentiment d'exterritorialité.

\footnotetext{
${ }^{10}$ La littérature des Peranakan, Chinois établis et assimilés depuis plusieurs générations en Indonésie et qui s'expriment en malais, fait à nouveau référence à la Chine à partir des années 1930 à la suite du conflit sinojaponais. Cf. Cl. SALMON, 1992 et Th. RIEGER, 1992.
} 
La perception d'exterritorialité a un effet technique assez simple : la vision des échanges et des relations au-delà des frontières, donc la possibilité de dessiner des réseaux, d'imaginer, de concevoir des relations qui les outrepassent. Dans un sens, cela permet plutôt de lier un point à un autre (une localité à une autre) que de passer d'un ensemble à un autre (d'un pays à un autre). Dans ce double cadre spatial de référence, le local et l'international, la multiplicité des réseaux ouvre l'éventail des choix.

\section{Primauté des relations humaines}

Dans la mesure où a été fait le choix, même inconscient, de persévérer en son être au sein de cette a-topie, il a fallu se projeter non pas sur un territoire précis et circonscrit, tel celui des nations sédentaires, mais dans un en-soi supraterritorial ; ne plus compter sur le " pays », entité humaine et territoriale, mais sur le groupe, le corps social. L'enracinement est détourné du sol vers l'âme, l'identité, l'être du groupe. Du couple d'éléments identitaires fondamentaux des groupes que constituent le territoire et l'être, c'est le second terme qui est progressivement privilégié par la diaspora.

Ce glissement de l'identification à l'entité " nation-territoire » vers l'identification à l'entité « communauté-ethnie » explique la puissance des liens de solidarité, fondés sur le sentiment d'interdépendance. L'empathie vers les proches remplace l'attachement au territoire. C'est vraisemblablement pour cela qu'il y a chez les membres de la diaspora comme une conscience diffuse, un pari, de faire confiance à quelque chose qui émanerait d'eux-mêmes, de leur être tel qu ' ils en ont conscience, d'eux rassemblés, comptant sur leurs propres forces, plutôt que de s'adresser à une construction supérieure, surplombante, organisatrice et qui leur échapperait, telle l'État. L'organisation est de type communautaire, plutôt que nationaleterritoriale. Mais ce sont bien les conditions de la migration, son volume et l'échelle de sa dispersion qui organisent la diaspora plutôt qu'une essence communautariste qui lui préexisterait. C'est parce qu 'à mesure qu 'elle se constitue en diaspora elle trouve un intérêt général plus grand dans cette forme d'organisation que dans le système national-territorial qu'elle la privilégie progressivement.

La mise en place de réseaux s'appuyant sur des personnes identifiées comme semblables en vue d'établir des relations ressortit à cette même logique d'éloignement et de défiance vis-àvis de l'extracommunautaire. C'est ainsi que font les entreprises pour recruter leur maind'oeuvre et asseoir leur financement : les flux financiers chinois vers les États-Unis, d'où qu'ils viennent, vont de préférence dans les villes où il y a déjà des chinatowns ${ }^{11}$.

\section{Valorisation de l'exterritorialité}

Dans le mouvement de dispersion de la diaspora, le maintien de l'identité à travers l'identification charnelle, physique à la terre d'origine n'est plus possible. L'identification à un peuple, à une culture, à un " être ensemble » prend le pas sur les rapports physiques. On intellectualise l'identité, l'acte d'identification ; le rapport à la culture se présente comme substitut au rapport à la mère-patrie, entité humaine et territoriale.

\footnotetext{
${ }^{11}$ Cf. J. LIN (1992). Sur les effets de ces investissements sur la Chinatown de New York, voir J. LIN (1990) et M. ZHOU (1993).
} 


\section{La culture et le corps social comme territoire}

Le corps social devient le territoire en tant qu'il permet de fixer l'identité individuelle et collective, d'où l'importance attachée à la culture. D'autant que « cette culture [est] si pérenne depuis trente siècles qu 'elle finit par se passer de référence territoriale » (P. Trolliet, 1994). Mais, de plus en plus, c'est un attachement à une culture de diaspora ${ }^{12}$, sous des formes très variables dans l'espace et dans le temps, comme le montre l'acculturation quasi totale puis la resinisation des Peranakan d'Indonésie et des Baba de Singapour; ou, inversement, quand de forts particularismes dans un lieu sont suivis d' une assimilation après migration dans un autre lieu. La culture de la diaspora s'élabore à travers l'encouragement de valeurs transnationales et la production de signes qui soulignent le caractère transnational de la migration chinoise ${ }^{13}$. Un bon exemple en est donné par l'appellation des banques à Hong Kong, dont certaines font explicitement référence aux Chinois d'outremer : Huachiao ${ }^{14}$ Commercial Bank, Overseas Chinese Banking Corporation, Overseas Chinese Commercial Bank, United World Chinese Commercial Bank, Overseas Union Bank, United Overseas Bank, United Chinese Bank, Overseas Trust Bank, Nan Yang ${ }^{15}$ Commercial Bank, Bank of Asia, International Commercial Bank of China, Far East Bank and Trust Company, etc. Un des vecteurs de cette formation d'une culture de la diaspora est l'information, qui permet la prise de conscience. L'importance numérique de la presse publiée par les Chinois d'outre-mer est d'ailleurs remarquable : pour la seule France, deux quotidiens et plusieurs périodiques sont publiés à Paris ; en Californie comme sur la côte orientale des États-Unis, existent plusieurs quotidiens et de nombreux périodiques. La transnationalité de ces publications apparaît dans leur titre à travers des référents continentaux, transcontinentaux, internationaux, voire mondiaux ; son affichage est un encouragement, et le signe qu'elle est positivement perçue par ceux auxquels il s'adresse.

Cette culture qui se met en place permet en retour de consolider la diaspora en lui fournissant des bases et des arguments idéologiques. C'est ainsi que les études sur les migrations chinoises, après avoir été longtemps consacrées aux implantations dans chacun des différents pays d'immigration, tendent aujourd'hui à envisager ces migrations dans leur dimension internationale et soulignent leur caractère diasporique. Par exemple, le colloque sur The Legal, Political and Economie Status of the Chinese in the Diaspora qui s'est tenu en novembre 1992 à San Francisco, l'une des villes symboles de la migration chinoise, a rassemblé près de 250 chercheurs et universitaires dont plus de 90 \% étaient d'origine chinoise et venaient de plus de 40 pays différents ; ce fut là une expression manifeste du caractère diasporique de la migration chinoise, en même temps qu'une affirmation et une forme de légitimation intellectuelle de la diaspora, à usage externe et, plus encore, à usage interne, argumentaire et justificatoire.

\section{La diaspora comme réponse aux injonctions identitaires}

Cette culture de la diaspora peut être considérée comme une réponse à la question, à première vue insoluble, à laquelle est confronté tout groupe migrant dès lors que son séjour perdure :

\footnotetext{
${ }^{12}$ Pour une approche différente de la culture de diaspora, R. GALLISSOT (1992), W. DRESSLER-HOLOHAN (1992), N. LAPIERRE (1992).

${ }^{13}$ Sur les relations entre culture chinoise traditionnelle et activités économiques : M. DEVERGE (1987), J. P. HASSOUN (1992), G. HOFSTEDE et M. HARRIS BOND (1988), S.K. LAU et H.C. KUAN (1988), C. PAIX et M. PETIT (1989), S.G. REDDING (1993), S.L. WONG (1988).

${ }^{14}$ C'est-à-dire Chinois d'outre-mer.

${ }^{15}$ Nan Yang : mers du Sud.
} 
intégration-assimilation ou préservation de l'identité collective. À divers moments, selon la conjoncture des pays d'accueil, les individus et les groupes sont sommés de dire qui ils sont et, dans l'esprit de ceux qui les interrogent, à quel pays ils font allégeance: celui d'origine ou celui d'accueil. D'où la fréquente accusation de double allégeance ${ }^{16}$ car ces injonctions sont le plus souvent faites dans le cadre d'une problématique de l' État-nation. Pour la diaspora la question posée est la suivante: comment s'intégrer individuellement et collectivement tout en conservant son identité individuelle et, surtout, collective?

Dans une certaine mesure, la réponse est géographique : ailleurs est ici car ici est ailleurs, ce qui exprime la difficulté à vouloir tenir l'espace en un lieu, à être immobile. Du point de vue de la diaspora, ce n'est que dans la fluidité et le mouvement que l'on peut y parvenir. Les inversions géographiques que ces jeux induisent, ces circularités topographiques, ces boucles topologiques illustrent bien la difficulté de l'être spatial des diasporas, l'incertitude à se situer. Mais ces jeux montrent également une tentative d'unification des lieux. " Ailleurs » est convoqué " ici », mais, dans le même temps, il doit rester ailleurs parce qu'ainsi il garantit d'être ici. Dans la réponse à la question " pouvez vous être d'ici en étant d'ailleurs ? » la diaspora, à travers le processus d'exterritorialisation, fait disparaître la préposition $d^{\prime}$ en répondant que l'on peut être ici en étant ailleurs. La réponse est un pari d'ubiquité, et suppose le talent ubiquiste constitutif de la diaspora.

\section{Conclusion : non-lieu et utopie}

L'exterritorialisation implique une prise de conscience de la configuration spatiale de la diaspora, puis une positivation de cette configuration à travers le développement d'une culture de la diaspora. Ce processus conduit à optimiser les ressources spatiales de la diaspora, que représentent la multipolarité de la migration et l'interpolarité des relations ; ce qui, en retour, renforce le caractère diasporique. Une société est possible en dernière analyse, disait Louis Wirth (1936), parce que les individus qui la composent véhiculent dans leur tête une sorte d'image de cette société. Dans le cas de la migration internationale chinoise, c'est parce que se met en place une vision de soi à l'échelle internationale que celle-ci se voit progressivement comme une diaspora au sens morphologique. Les formes objectives rendent possible cette représentation qui, en retour, influencent ces formes.

La position d'exterritorialité, la perception de ce non-lieu, est donc une étape indispensable comme intellectualisation du fait morphologique de la diaspora pour établir et améliorer son fonctionnement en diaspora. D'une part, elle a un effet utilitaire, car elle permet de percevoir les avantages de cette particulière disposition pour développer ses relations. D'autre part, elle permet de parer aux injonctions identitaires et au procès de double allégeance. Ajoutons qu'elle a un effet compensatoire dans la mesure où elle permet de soigner la mauvaise conscience de la diaspora, souvent coupable d'abandon aux yeux de la société d'origine ; et, plus fondamentalement, de sublimer cette amputation d'être sans territoire.

Dans une certaine mesure, la diaspora est une nouvelle forme sociale, expression des nouvelles formes de rapports que la société entretient à l'espace, et qui annoncent peut-être la fin des territoires : " Verra-t-on apparaître un jour prochain, issues des diasporas, des sortes de nations multinationales susceptibles de prendre en défaut - de déranger ou de stimuler selon

\footnotetext{
${ }^{16}$ La question est également posée par la Chine avec en arrière-plan la même accusation, comme en témoignent les fréquents changements d'attitude de la « mère patrie » vis-à-vis des émigrés chinois au cours de l'histoire. Sur l'évolution des relations entre les Chinois d'outre-mer et la Chine, cf. G. WANG (1991 et 1992) et G. ZHUANG (1992).
} 
les cas - les nations campant sur leurs habitus ? »(C. Benayoun et A. Médam, 1992). L' atopie dans laquelle essaie de se tenir la diaspora lui permet de prononcer un non-lieu au procès de la double allégeance et de concevoir puis de donner corps à cette utopie qui consiste à rêver de soi-même dans une vie hors du territoire et à vouloir être dans le même temps au Monde et au pays.

\section{Références}

BAO Daniel (1992). « No hay sangre fresca aqui : the Chinese community in Montevideo, Uruguay». Communication à la conférence internationale Luo Di Sheng Gen : The Legal, Political and Economic Status of Chinese in the Diaspora, November 26-29, Miyako Hotel, San Francisco. University of California at Berkeley.

BENAYOUN Chantal, MÉDAM Alain (1992). « L'actualité des diasporas ». Actes du colloque Intelligence de l'Europe, Lyon, juillet.

BRUNEAU Michel (1994). « La diaspora pontique et ses territoires ». L'Espace géographique, $n^{\circ} 2$.

CHAN Kwok-Bun et al. (eds) (1994). Asian Transmigration. Singapore : Prentice Hall (à paraître).

CHARBONNIER Jean (1987). « Les Chinois de la diaspora ». Études, n³67.

CLAIRMONT Frédéric F. (1993). " L’argent de la diaspora, nerf de la croissance ». Le Monde diplomatique, avril.

CUSHMAN Jennifer, WANG Gungwu (eds) (1988). Changing Identities of the Southeast Asian Chinese since World War II. Hong Kong : Hong Kong University Press, 344 p.

DENNERY Étienne (1930). Foules d'Asie. Paris : Armand Colin, 240 p.

DESCHAMPS René (1908). La main-d'oeuvre en Indochine et l'immigration étrangère. Poitiers: Université de Poitiers, Thèse pour le doctorat de sciences politiques et économiques.

DEVERGE Michel (1987). « Confucianisme et succès économique à Taiwan ». Études, nº367.

DRESSLER-HOLOHAN W. (1992). "Culture minoritaire, culture nationale et culture de diaspora ». Informations sur les sciences sociales, vol. $31, \mathrm{n}^{\circ} 2$.

GALISSOT René (1992). « Pluralisme culturel en Europe : identités nationales et identité européenne. De l'intellectuel métis au métissage culturel de masse ». Informations sur les sciences sociales, vol. $31, n^{\circ} 1$.

HALBWACHS Maurice (1971). La Topographie légendaire des Évangiles en Terre Sainte. Paris: Presses universitaires de France, 171 p.(1 ${ }^{\text {ere }}$ éd., 1941).

HASSOUN Jean-Pierre (1992). " Pratiques religieuses et entreprises Chinoises à Paris, un paysage favorable ». Revue européenne des migrations internationales, vol.8, n³.

HIRSCHMAN Charles (1988). " Chinese identities in Southeast Asia : alternative perspectives ». In Jennifer CUSHMAN and WANG Gungwu (eds), Changing Identities of the Southeast Asian Chinese since World War II. Hong Kong : Hong Kong University Press, 1988, 344 p.

HOFSTEDE Geert, HARRIS BOND Michael (1988). « The Confucius connection: from cultural roots to economic growth ». Organizational Dynamics, vol. 16, $n^{\circ} 4$.

LAPIERRE Nicole (1992). " Le modèle de la diaspora juive en Europe ». Informations sur les sciences sociales, vol. $31, \mathrm{n}^{\circ} 1$.

LAU Siu-Kai, KUAN Hsin-Chi (1988). The Ethos of the Hong Kong Chinese. Hong Kong : The Chinese University Press, $217 \mathrm{p}$.

LE CALLOCH Bernard (1977). «La diaspora chinoise en France ». Acta geographica, $3^{\mathrm{e}}$ série, $\mathrm{n}^{\circ} 30$.

LEW Roland (1993). « Les espoirs du capitalisme en Chine ". Le Monde diplomatique, avril.

LIN Jan (1990). " Polarized development in the 'world city' : the chinese enclave of New York city". Communication au colloque international sur le logement, Housing debates - Urban challenges, 36 juillet, Paris.

LIN Jan (1992). «Flux de main-d'oeuvre et de capitaux chinois vers les États-Unis ". Revue européenne des migrations internationales, vol. $8, \mathrm{n}^{\circ} 3$.

LYMAN Stanford M. (1974). Chinese Americans. New York : Random House.

MA MUNG Emmanuel (1991). "Le développement de l'entreprenariat chez les Chinois : facteur d'autonomisation de la communauté ? " Communication au colloque international organisé par l'ARIC (Association pour la Recherche InterCulturelle), Ministère de la Recherche, Paris, 14-16 octobre. 
MA MUNG Emmanuel (1992 a). «L'expansion du commerce ethnique : Asiatiques et Maghrébins en région parisienne ». Revue européenne des migrations internationales, vol. $8, \mathrm{n}^{\circ} 1$.

MA MUNG Emmanuel (1992 b). « Dispositif économique et ressources spatiales: éléments d'une économie de diaspora ». Revue européenne des migrations internationales, vol. 8, $\mathrm{n}^{\circ} 3$.

MA MUNG Emmanuel (1994). « L'entreprenariat ethnique en France ". Sociologie du travail (à paraître).

MA MUNG Emmanuel, ABDULKARIM Amir (1991). « China y Libano, una misma diaspora ? " El País, jueves 20 de junio. Dossier spécial : Movimiento y Migración.

MA MUNG Emmanuel, SIMON Gildas (1990). Commerçants maghrébins et asiatiques en France. Paris: Masson, coll. « Recherches en géographie ", 138 p.

MÉDAM Alain (1991). Mondes juifs, l'envers et l'endroit. Paris : Presses universitaires de France, 192 p.

MON Ramon A. (1992). «Procesos de integración de la communidad china a la nación panameña ". In Convergencias étnicas en la nacionalidad panameña, ILDEA (Instituto Latinoamericano De Estudios Avanzados), Panama.

PAIX Catherine, PETIT Michèle (1986). « Itinéraires et stratégies d'une bourgeoisie, le cas de Singapour ". Strates, $\mathrm{n}^{\circ} 1$.

PAIX Catherine, PETIT Michèle (1989). « Espaces graphiques et pratiques de pouvoir : philosophies d'entreprises à Taiwan, préceptes politiques à Singapour ". Strates, $\mathrm{n}^{\circ} 4$.

POSTON Jr. Dudley L., YU Mei-Yu (1990). "The distribution of the overseas Chinese in the contemporary world ». International Migration Review, vol. 24, p. 480-508.

PURCELL Victor (1965). The Chinese in Southeast Asia. London : Oxford University Press.

REDDING S. Gordon (1993). The Spirit of Chinese Capitalism. New York : de Gruyter, 267 p.

RIEGER Thomas (1992). " La guerre sino-japonaise dans la littérature indonésienne ». In SALMON Claudine, « Le moment "sinomalais" de la littérature indonésienne ». Archipel, n¹9, 184 p.

SALMON Claudine (1988). " Post-war fiction in Chinese as a mirror of political, social and cultural changes in Southeast Asia». In CUSHMAN Jennifer, WANG Gungwu (eds), Changing Identities of the Southeast Asian Chinese since World War II. Hong Kong: Hong Kong University Press, $1988,344 \mathrm{p}$.

SALMON Claudine (dir.) (1992). « Le moment "sino-malais" de la littérature indonésienne ». Archipel, $\mathrm{n}^{\circ} 19,184 \mathrm{p}$.

SIU Lok (1992). " Apple king Nicaragua, Willie Siu : remembering biographies ». Communication à la conférence internationale Luo Di Sheng Gen : The Legal, Politieal and Eeol/omie Status of Chil/ese in the Diaspora, November 26-29, 1992, Miyako, Hotel, San Francisco. University of California at Berkeley.

SKELDON Ronald (1992). « International migration within and from the East and Southeast Asian region : a review essay ». Asian and Pacific Migration Journal. Vol. l, $\mathrm{n}^{\circ} 1$.

TARRIUS Alain (1994). "Territoires circulatoires des migrants et espaces urbains ». Revue française de sociologie (à paraître)

The Economist (18 juillet 1992). « The overseas Chinese ».

Time (10 mai 1993).« The secret weapon ».

TROLLIET Pierre (1982). « Les Chinois en Asie du Sud-Est ». Hérodote, n 21.

TROLLIET Pierre (1987). « Réflexions sur la diaspora des Chinois ». Cahiers d'études chinoises, n6.

TROLLIET Pierre (1994). « Peut-on parler d'une diaspora chinoise ? ». L'Espace géographique, $n^{\circ} 2$.

TSAI Maw-Kuey (1968). Les Chinois au Sud Vietnam. Paris: Bibliothèque Nationale, Thèse de géographie, $293 \mathrm{p}$.

WANG Gungwu (1991). China and Chinese overseas. London: Times Academic Press, 312 p.

WANG Gungwu (1991). The Chineseness of China. Oxford: Oxford University Press, 354 p.

WANG Gungwu (1992). Communily and nation. Londres: Allen \& Unwin, 358 p.

WIRTH Louis (1936). Préface. in Karl MANNHEIM, Idéologie et utopie [Idéologie und Utopie, 1929]. Paris : Éd. Rivière, 1956, 234 p.

WONG Maria Lin (1989). Chinese Liverpudlians. Liverpool: Liver Press, 100 p.

WONG Siu-Lun (1988). Emigrants Entrepreneurs. Oxford : Oxford University Press, 244 p.

ZHOU Min (1993). Chinatown, the Socioeconomic Potential of an Urban Enclave. Philadelphie: Temple University Press, 328 p.

ZHUANG Guotu (1992). " Policies of China's government towards returned Overseas Chinese and dependants of the Chinese abroad (1949-1966). Communication à la conférence internationale Luo Di Sheng Gen: The Legal, Political and Economic Status of Chinese in the Diaspora, November 26-29, Miyako Hotel, San Francisco. University of California at Berkeley. 